$(\mathrm{n}=1)$, or low nasal NO, clinical phenotype and an affected sibling $(\mathrm{n}=3))$. There was no significant difference in age or gender composition between the 3 groups.

Results Patients with normal ultrastructure had significantly higher $\mathrm{FEV}_{1}$ and lower LCI, indicating milder disease. Those with ODA +/- IDA had a more normal LCI than those with microtubular defects (Figure 1), but similar $\mathrm{FEV}_{1}$.

Conclusions PCD patients with normal ultrastructure have the milder disease, and those with microtubular defects more severe. Differences were more apparent on LCI than $\mathrm{FEV}_{1}$, suggesting LCI may be more sensitive to worse distal small airway disease in PCD.

\section{P83 REMOTE PULMONARY FUNCTION TESTING - COMPUTER GAMING IN THE RESPIRATORY WORLD}

${ }^{1} \mathrm{C}$ Sharp, ${ }^{2} V$ Soleimani, ${ }^{2} \mathrm{~S}$ Hannuna, ${ }^{2} \mathrm{M}$ Camplani, ${ }^{2} \mathrm{D}$ Damen, ${ }^{3} \mathrm{~J}$ Viner, ${ }^{2} \mathrm{M}$ Mirmehdi, 1J Dodd. 'Academic Respiratory Unit, University of Bristol, Bristol, UK; ${ }^{2}$ Visual Information Laboratory, Faculty of Engineering, University of Bristol, Bristol, UK; ${ }^{3}$ Respiratory Physiology, North Bristol NHS Trust, Bristol, UK

\subsection{6/thoraxjnl-2015-207770.220}

Introduction Lung function testing by spirometry has remained unchanged for over 50 years, despite limitations including patient technique, discomfort, cost and training. Non-invasive, remote lung volume measurement is an alternative approach. This has been explored in the past using structured light, accelerometers and impedance pneumography, but these have often been costly and of limited validity.

We use a novel approach to remote assessment ( $\sim 2$ metres) using a 3D time-of-flight depth camera - similar to those found in many home gaming consoles. This pilot developmental data was generated from patients in a clinical setting.

Methods Patients were recruited from a general respiratory physiology laboratory. Spirometry was performed according to ATS/ ERS standards using an unmodified pneumotachograph (nSpire Health, Longmont, CO, USA). A Kinect V2 time-of-flight depth sensor (Microsoft, Redmond, WA, USA) was used to reconstruct 3D models of the subject's thorax to estimate volume-time and flow-time curves for both Forced and Slow Vital Capacity and their associated measurements (Figure 1, technical details in ${ }^{1}$ ).

These results were correlated with simultaneous recordings from the pneumotachograph, and error values calculated to assess the accuracy of the technique.

Results Data were available from 53 patients, with 40 having usable data. Mean age 62.8 yrs (SD 16.2), BMI of 26.8 (SD 5.5). $41.5 \%$ male. $54.7 \%$ of patients had obstructive lung diseases, and 28.4\% fibrotic lung disease. Mean FVC was 91.3\% predicted (SD 26.4\%), Mean FEV1 83.1\% (SD 28.9\%).

The model estimates were highly correlated with spirometric values for FVC $(\lambda=0.999)$, FEV1 $(\lambda=0.947)$, VC $(\lambda=$ 0.999), IC $(\lambda=0.997)$ and TV $(\lambda=0.962)$.

Univariate analysis demonstrated no patient characteristics predictive of discrepancy from spirometric values for FVC or VC.

Conclusions We describe a pilot data from the initial development of a new technique for non-invasively assessing lung volume and pulmonary function measurements. It correlates to within $30 \mathrm{ml}$ for FVC and $10 \mathrm{ml}$ for VC. This has a wide range of potential applications, including screening, home monitoring of respiratory disease, assessment of lung function in those unable to complete pneumotachygraphy and gating controls for radiological imaging techniques.
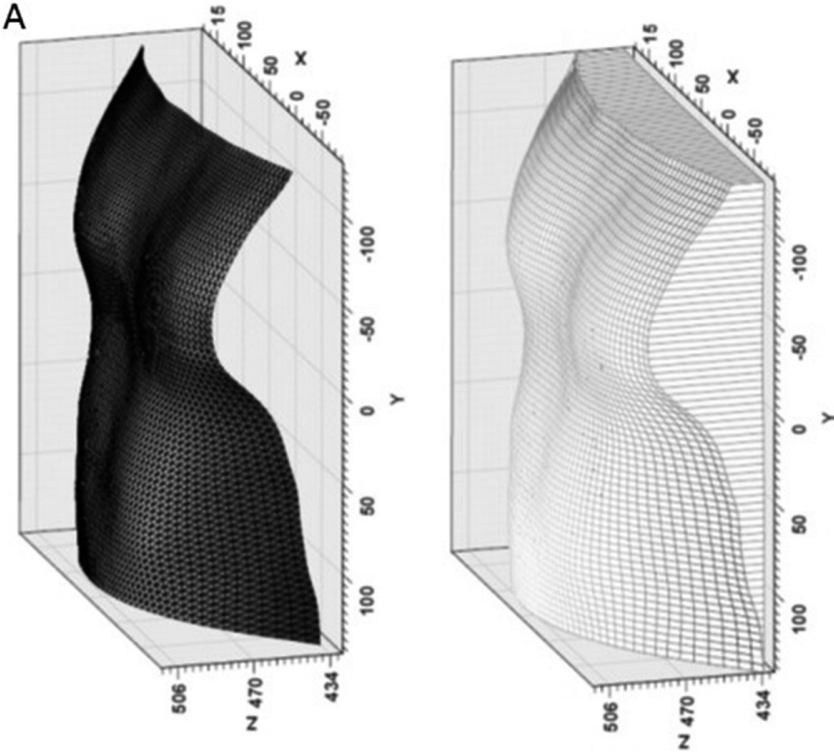

B
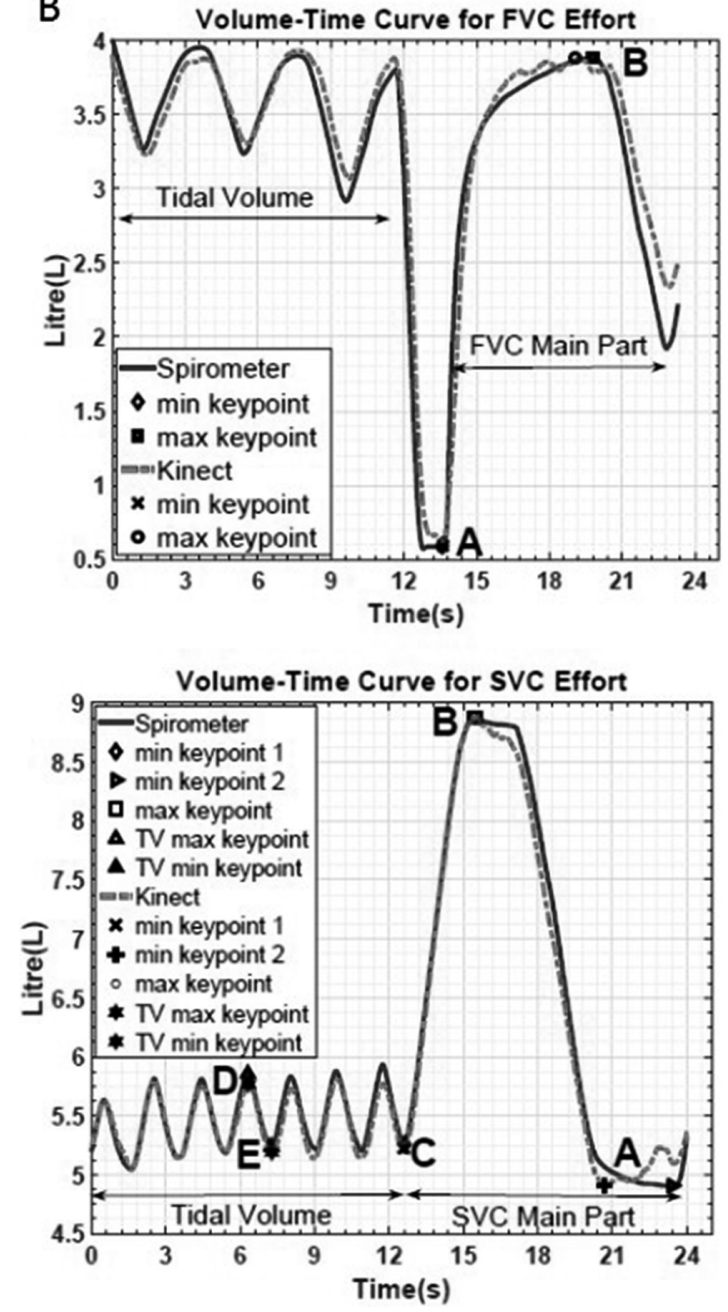

Abstract P83 Figure 1 (A) Reconstructed chest surface and volume estimation from reference plane. (B) Volume-time curves (FVC and SVC) comparing spirometer values and Kinect calculation

\section{REFERENCE}

1 Soleimani $\mathrm{V}$, Mirmehdi $\mathrm{M}$, Damen $\mathrm{D}$, et al. Remote pulmonary function testing using a depth sensor. Biocas 2015 


\section{P84 THE INSPIWAVE (TM) TRIAL ON ADULT HEALTHY VOLUNTEERS - INSIGHTS GLEANED FROM POSTURAL STUDIES}

${ }^{1} \mathrm{C}$ Zhang, ${ }^{2} \mathrm{P}$ Phan, ${ }^{3} \mathrm{D}$ Geer, ${ }^{2} \mathrm{C}$ Hahn, ${ }^{2} \mathrm{~A}$ Farmery. ${ }^{1} \mathrm{G} K T$ School of Medical Education, King's College London, London, UK; ${ }^{2}$ Nuffield Department of Clinical Neurosciences, University of Oxford, Oxford, UK; ${ }^{3}$ The Queen's College, University of Oxford, Oxford, UK

\subsection{6/thoraxjnl-2015-207770.221}

Introduction Current techniques used to monitor Functional Residual Capacity (FRC) present significant clinical limitations which often restrict patients to those who are conscious, cooperative and mobile. There is a clinical need for an operator - independent device that is able to simultaneously and continuously monitor FRC in mechanically ventilated patients, the morbidly obese and infants. The InspiWave ${ }^{\mathrm{TM}}$ is one such device which utilises a sinusoidal Nitrous Oxide $\left(\mathrm{N}_{2} \mathrm{O}\right)$ tracer gas technique to non - invasively monitor various parameters of cardiopulmonary function, including end tidal alveolar volume $\left(\mathrm{V}_{\mathrm{A}}\right)$ and $\mathrm{Bohr}$
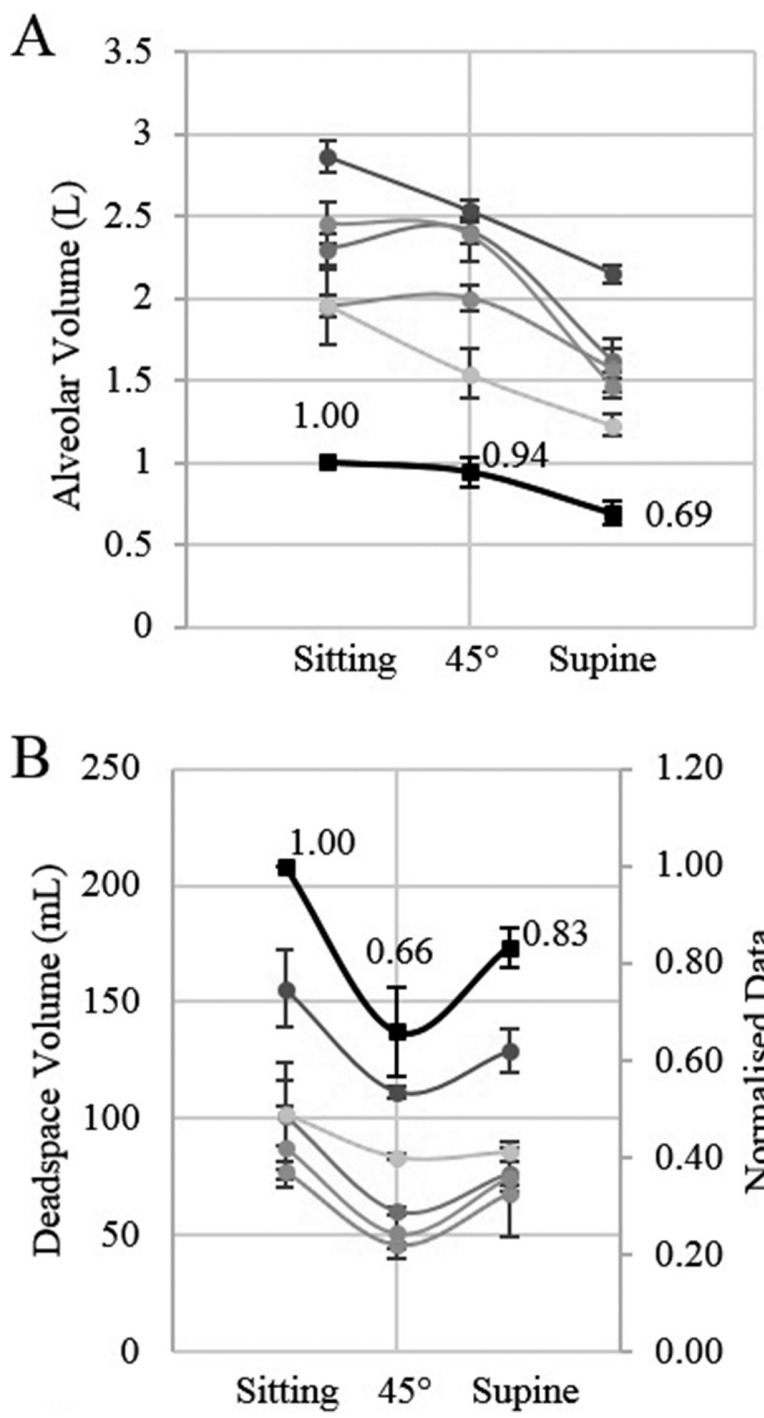

1.20

1.00

0.80

0.60

0.40

0.20

0.00

Abstract P84 Figure 1 Alveolar Volume (A) and Deadspace Volume (B) estimated by the device at three different postures. Averaged data of participants $(n=5)$ shown in traces with circle markers $(\bullet)$, error bars represent standard deviation. Normalised data relative to the upright sitting position plotted with square markers (ם), error bars represent $95 \%$ confidence interval deadspace volume $\left(\mathrm{V}_{\mathrm{D}}\right)$. This study evaluates the performance of the InspiWave ${ }^{\mathrm{TM}}$ in estimating lung volume using two unique postural change protocols in healthy volunteers.

Method 5 subjects were asked to adopt 3 stationary postures (sitting upright, supine, $45^{\circ}$ elevation) using a tilt table. 21 subjects adopted a different protocol, with a single transition phase between sitting and supine.

Results In the first protocol $(n=5), V_{A}$ estimations were consistently smaller at supine than at sitting (31\%). At $45^{\circ}$ elevation, $V_{D}$ estimations were the smallest of all three postures. Comparison of absolute flow signal $\left(\Delta \int\right.$ Flow $\left.d t\right)$ data at the point of postural transition with $\mathrm{V}_{\mathrm{A}}$ estimations by the device $(\mathrm{n}=21)$ yielded a Pearson's correlation $(r)$ of $0.453(p<0.05)$.

Conclusion Estimations by the InspiWave ${ }^{\mathrm{TM}}$ demonstrated acceptable correlation between absolute flow signal change and device estimation, suggesting good accuracy. FRC changes by approximately $25 \%$ from sitting to supine, due to added pressure from abdominal contents on the diaphragm when gravity shifts with postural change. ${ }^{1}$ Data from this study closely mirrored this value (31\%), demonstrating a good degree of device performance. $V_{D}$ estimations were smallest at $45^{\circ}$ elevation of the three postures which may be due to airways being kept more open, consistent with respiratory benefits of the $45^{\circ}$ "beach chair position”. Postural studies provide an alternative over conventional comparative studies with reference techniques (such as body plethysmography) in assessing device performance, when comparing variables which are not entirely equivalent.

\section{REFERENCE}

1 Ibanez J, Raurich JM. Normal values of functional residual capacity in the sitting and supine positions. Intensive Care Med. 1982;8(4):173-177

\section{P85 A COMPOSITE INDEX OF SATURATION AND DISTANCE WALKED DURING A 6-MINUTE WALK TEST (6MWT): A RETROSPECTIVE METHODOLOGICAL COMPARISON}

R Brown, AH Kendrick. Department of Respiratory Medicine, University Hospitals, Bristol, UK 10.1136/thoraxjnl-2015-207770.222

Background The 6MWT is a simple, reproducible test of exercise performance. Lettieri et al. $^{1}$ proposed a composite index of the product of the lowest oxygen saturation $\left(\mathrm{SpO}_{2}\right)$ and distanced walked - the distance-saturation product (DSP). Pimenta et al. ${ }^{2}$ calculated the differences between actual $\mathrm{SpO}_{2}$ and $100 \%$ every two seconds, and produced the desaturation-distance ratio (DDR-1). Ijiri et al. ${ }^{3}$ simplified Pimenta's methodology, using the $\mathrm{SpO}_{2}$ at the end of each minute (DDR-2).

Aim To compare the different methods of calculating the composite index.

Methods We retrospectively analysed 48 6MWT datasets, obtained using standard methodology. $\mathrm{SpO}_{2}$ was recorded every second using a Minolta 300i pulse oximeter and analysed using Visi-Download (Stowood Scientific, Oxford). The DSP and DDR's was calculated by each method. Additionally, data using Pimenta's method, was calculated using one second data intervals (DDR-3). Data are given as median (IQR).

Results $41 / 48$ patients were male. Group age was 63 (53 to 73) years, $\mathrm{FEV}_{1}$ \%pred $68.4 \%$ (41.3 to 82.3 ), distance walked $390 \mathrm{~m}$ (321.3 to 477.5), baseline $\mathrm{SpO}_{2}$ 95\% (93 to 96) and decline in $\mathrm{SpO}_{2}$ was $-4 \%$ (-2 to -8$)$. DSP was 324.6 (278.8 to 419.4$) \mathrm{m} \%$, DDR-1 was 8.90 (4.98 to 13.27), DDR-2 was 16.83 (9.14 to 24.8) and DDR-3 was 8.90 (4.99 to 13.66). Bland-Altman 